Int. J. Curr. Res. Med. Sci. (2017). 3(3): 125-130

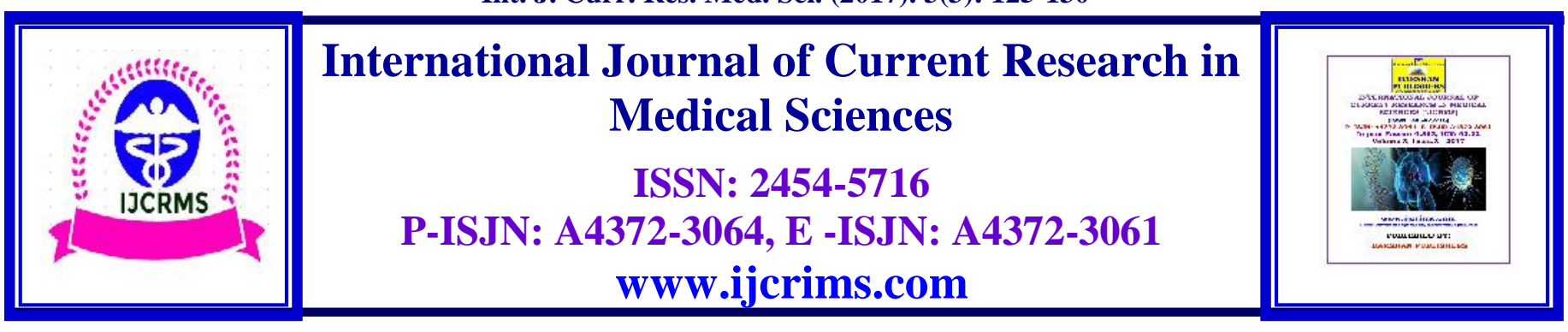

\title{
Prevalence of Oral Thrush Yeasts among School Children with special emphasis of Fluconazole antifungal drug
}

\author{
Dr R.Venkatajothi \\ Former, Senior Lecturer, Department of Microbiology, Faculty of Medicine \\ AIMST University, Kedah Darul Aman, Malaysia. \\ *Corresponding author:drrvjothi10@gmail.com
}

\begin{abstract}
The fungus Candida albicans commonly causes of Oral thrush and when the fungus begins to grow uncontrollably, an infection can develop in the mouth. Oral thrush most often occurs in infants and children. It causes white bumps to form on the inner cheeks and tongue. This condition is also known as Oropharyngeal Candidiasis. The aim of the study was to analysis the prevalence of Oral thrush yeasts among School children and to carry out their antifungal susceptibility pattern to Fluconazole antifungal agent. A total number of 325 healthy school children (200 Boys and 125 girls) from Luqman Islamic Seminary Secondary School in rural area of Dar-Es-Salaam in Tanzania were sampled for the occurrence of Oral thrush yeasts. The children were aged between 5 to 12 years old. Candida yeasts were identified by using Sabouraud Dextrose Agar media and Antifungal susceptibility testing was performed for the isolates of Candida using disc diffusion method on Mueller Hinton agar media. Among of 325 School children sampled, $42.0 \%$ isolates were found to be Candida yeasts positive and $58.0 \%$ isolates were non Candida species. Further examination of the positive cases showed that Candida albicans occurred as $46 \%$ and Non Candida albicans species was 54\%. Antifungal susceptibility was done for 62 Candida albicans isolates. Among of them $81 \%$ isolates of Candida albicans showed sensitive to Fluconazole, followed by $16 \%$ isolates showed resistance and 3\% isolates were dose dependent susceptibility. The results showed that the infection occurred more in younger children below 8 year old than in older children. Further, the comparison result showed that male children have more susceptible to Candida albicans infections than the female children. The problem posed by Oral thrush to the children was highlighted and control measure recommended to reduce incidence and spread.
\end{abstract}

Keywords: Candida, Yeast, Candida albicans, Fluconazole

\section{Introduction}

Candidiasis is the commonest fungal disease found in humans affecting skin, mucosa, nails and internal organs. Oral thrush is a superficial fungal infection of the mucous membrane characterized by white adherent patches of pseudomycelium which often involves lesions, sores, fissures and ulcers in the mouth which could either be acute or chronic. Oral thrush is typically a mild infection 
that rarely causes complications and it is mainly affect small children and elderly persons ${ }^{2,7}$. Oral thrush is mainly caused by $\boldsymbol{C}$. albicans followed by $\boldsymbol{C}$. tropicalis, $\boldsymbol{C}$. parapsilosis and other species of Candida ${ }^{13}$.

Candida species is commonly known as Opportunistic pathogens which reside in the human buccal cavity are the most common causes of Oral thrush. The condition can be problematic for those with weakened immune systems and in weak immune system, the fungus may enter bloodstream and spread throughout the body. This can eventually cause problems in various body structures, including the brain, heart and liver. Oral thrush is also very common in HIV/AIDS patients ${ }^{7}$.

They live in the mouth as normal commensals but can cause some infection when there is amendment in their environmental conditions. Candida species can also be isolated in good quantity from the mouth of both healthy subjects who have no clinical evidence of Candida infection previous. It may cause systemic involvement and can sometimes be fatal. Other types of fungi commonly causes of Oral thrush are Aspergillus sp. and Cryptococcus neoformans ${ }^{11}$. Candidial infection is associated with many risk factors including age, pregnancy, poor oral hygienic, diabetes, long term intravenous therapy, long term antibiotic use and immunosuppressive therapy ${ }^{6}$ etc.
Azole group of drugs such as Fluconazole, Itraconazole, Miconazole and Ketoconazol are commonly used in the treatment of various types of Candidal infections, however their prolonged use has led to the development of drug resistance in C.albicans and other species. Azole resistance is seen more commonly in Non Candida albicans spp. compared to C.albicans ${ }^{1,5}$.

Increasing emergence of drug resistance to azole groups, the identification and speciation of Candida isolates must be implemented in routine culture work. Antifungal susceptibility pattern helps in guiding the physician in choosing the appropriate antifungal drug and thus prevent therapeutic failures. This occurrence, if high, will call attention to the oral health of school children in this area of the country.

\section{Objectives of the Study}

The objective of the study was to analysis the prevalence of Oral Thrush Yeasts among School Children and to carry out their antifungal susceptibility pattern to Fluconazole antifungal agent.

\section{Materials and Methods}

The total study population was 325 (200 Boys and 125 Girls) Luqman Islamic Seminary Secondary School children in rural area of Dar-Es-Salaam in Tanzania were taken up for Oral Thrush identification. The children were aged between 5 12 years old (Table $\mathbf{1}$ ).

Table 1. Age and Sex wise Distribution of School children in study case $(\mathbf{n}=325)$

\begin{tabular}{|c|c|c|c|}
\hline S. NO & $\begin{array}{c}\text { Student Age } \\
\text { Group }\end{array}$ & $\begin{array}{c}\text { Total Boys } \\
\mathbf{n = 2 0 0}\end{array}$ & $\begin{array}{c}\text { Total Girls } \\
\mathbf{n = 1 2 5}\end{array}$ \\
\hline $\mathbf{1 .}$ & $5-6$ & 52 & 44 \\
\hline $\mathbf{2 .}$ & $7-8$ & 49 & 31 \\
\hline $\mathbf{3 .}$ & $9-10$ & 45 & 22 \\
\hline $\mathbf{4 .}$ & $11-12$ & 54 & 28 \\
\hline
\end{tabular}


The samples were collected by using sterile throat swabs. A questionnaire was used to obtain information on Age, Sex of the children and socioeconomic status of the children's parents. The isolates were considered significant by Direct wet mount, Gram stain, growth on culture, and acid \& gas production in sugar fermentation tests using Glucose, Maltose, Dextrose, Sucrose and Lactose in $2 \%$ concentration with Andred's indicator and Durham's tube test ${ }^{3,8}$.

A sterile throat swabs was carefully placed the mouth each of the students and the tongue, teeth, soft and hard palate were swabbed. The throat swab was removed and placed in a sterile container. All the samples were inoculated on already prepared Sabaroud's Dextrose Agar (SDA) slants supplemented with chloramphenicol and incubated at $37^{\circ} \mathrm{C}$ for $24-48 \mathrm{hrs}$. Any growth found on SDA slope was processed for identification. The macroscopic features of the growth were opaque pasty, smooth, glaborous, membranous texture often with sweet odour.

The fungus growth was confirmed by observing characteristic budding with pseudohyphae in Gram stain. Distinctive colonies had the characteristic yeast smell. After 3 days at room temperature, Candida albicans appeared as cream coloured, smooth colonies with pseudomyceluim growing into the agar. The yeast like colony growth on culture showing budding yeast cells in Gram's staining method were confirmed as Candida by negative Urease test. Germ tube test was performed on all Urease negative yeast isolates for presumptive identification of C. albicans $^{12}$.

Germ Tube test: About $0.5 \mathrm{~mL}$ of human blood serum was poured into a small test tube and each isolate inoculated into the tube using a sterile wire loop. Incubation was in a water bath for $24 \mathrm{~h}$. A drop of the serum yeast culture was transferred unto a glass slide using a Pasteur pipette and covered with a cover slip. Examination for germ tubes or blastospores was done under the X40 objective.

The presence of sprouting yeast cells was a positive identification for $\boldsymbol{C}$. albicans. The non C. albicans yeasts were identifying by differentiation was done using the ability to ferment various sugars and also the carbon assimilation test 9,10 . Antifungal susceptibility testing was performed for all the isolates of C. albicans using disc diffusion method on Mueller Hinton agar media. The commercially available antifungal discs Fluconazole $(10 \mathrm{~g})$, were used and zones of inhibition were measured after $24-48$ hours incubation at $37^{\circ} \mathrm{C}$.

\section{Results and Discussion}

Out of 325 school children sampled, 135 (42.0\%) isolates were found to be Candida yeasts positive and $190(58.0 \%)$ isolates were non Candida species (Table 2). Further examination of the positive cases showed that, Candida albicans occurred as $62(46 \%)$ isolates and Non candida albicans species was 73 (54\%) (Table 3).

Table 2. Distribution of Candida isolates among case study

\begin{tabular}{|c|l|c|c|}
\hline S. No & \multicolumn{1}{|c|}{ Contents } & Total numbers & P\# (\%) \\
\hline 1. & Total case study & 325 & $100 \%$ \\
\hline 2. & Candida yeasts positive & 135 & $42 \%$ \\
\hline 3. & non Candida species & 190 & $58 \%$ \\
\hline
\end{tabular}

Table 3. Distribution of Candida albicans among case study

\begin{tabular}{|c|l|c|c|}
\hline S. No & \multicolumn{1}{|c|}{ Contents } & Total numbers & Percentage (\%) \\
\hline 1. & Total number of Candida Isolates & 135 & $100 \%$ \\
\hline 2. & Candida albicans & 62 & $46 \%$ \\
\hline 3. & Non candida albicans & 73 & $54 \%$ \\
\hline
\end{tabular}


Thus, Candida albicans appeared to be the principal agent of Oral thrush in children in the schools sampled. This agreed with the work of BurdfordMason and Willoughby who found that this organism was the chief causative agent of oral thrush in children ${ }^{4}$.
Antifungal susceptibility was done for 56 Candida albicans isolates. Among of them 50 (81\%) isolates of Candida albicans showed sensitive to Fluconazole anti fungal agent, followed by $10(16 \%)$ isolates showed resistance and $2(3 \%)$ isolates dose dependent susceptibility (Table 4).

Table 4. Antifungal susceptibility pattern of Candida albicans to Fluconazole antifungal drug $(\mathbf{n}=62)$

\begin{tabular}{|c|c|c|}
\hline $\begin{array}{c}\text { Sensitive } \\
\text { Percentage } \\
(\%)\end{array}$ & $\begin{array}{c}\text { Susceptible dose } \\
\text { dependent } \\
\text { Percentage } \\
(\%)\end{array}$ & $\begin{array}{c}\text { Resistant } \\
\text { Percentage } \\
(\%)\end{array}$ \\
\hline $81 \%$ & $3 \%$ & $6 \%$ \\
\hline
\end{tabular}

Many studies were undertaken to find the development of resistance to antifungal agents among Candida species and in the present study $16 \%$ resistance to fluconazole was observed. This study highlights the need for rapid and precise identification of Candida isolates to species level for effective treatment and management strategies and also identification of rising anti-fungal resistant to most azole group of drugs.
In this study results also showed that the infection occurred more in younger School children below 8 year old than in older children. In addition to, the comparison result showed that male children had $58 \%$ of Candida and $63 \%$ of Candida albicans infections and female children had $42 \%$ of Candida and $37 \%$ of Candida albicans infection (Figure 1 \& 2). Hence, male children have most prone to Oral thrush infection than the female children.

\section{Distribution of Candida Infection among School Children}

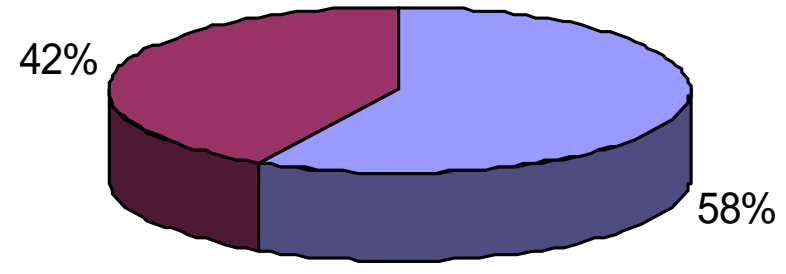

$\square$ Boys $\square$ Girls 
Figure 2:

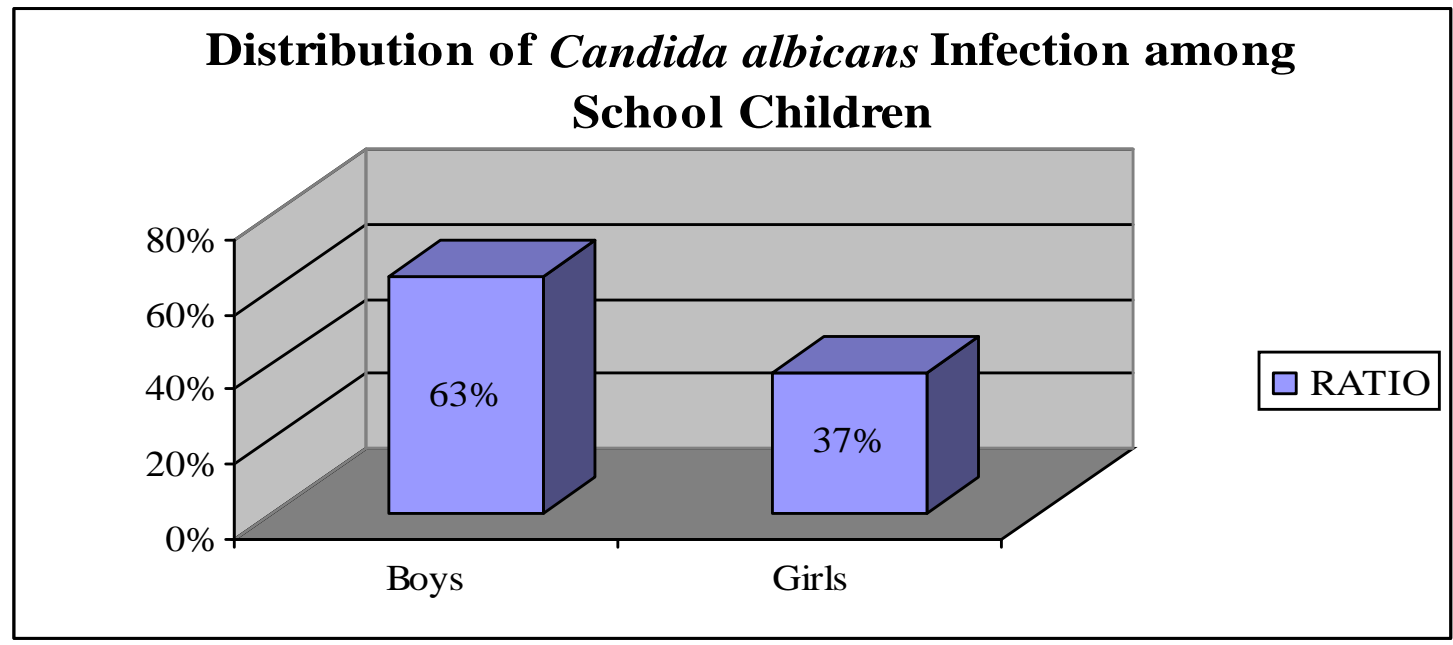

\section{Conclusion}

This study showed high prevalence of Candida albicans was mainly responsible for Oral thrush among children especially those below 8 years old. Further, the comparison result showed that male children have most susceptible to Oral thrush infections than the female children. Oral thrush poses a vital problem in children and as such its incidence needs to be reduced and the affected children taken care. Oral hygiene education was one of the important recommendations to the teachers for the oral welfare of the children and also children should be discouraged from the common use of cups and cutlery in schools to avoid cross infection. Then there is the occasional use of mild antiseptic mouthwash. When the children infected, antifungal medicines such as Fluconazole or other orally active azole derivatives should be used to treat the children.

\section{References}

1. Agarwal J, Seema B, Mallik G K, Jain A. Trends in neonatal septicemia: Emergence of non-albicans Candida. Indian Pediatrics 2004; 41:712-15.

2. Arendorf, T.W. and D.M. Walker, 1999. Oral candidal populations in health and diseases. Brit. Dent. J., 147: 267272.

3. Barnett, J.A., R.N. Payne and D. Yarrow, 1996. Yeast Characteristics and Identification. 2nd Edn., Cambridge University Press, Cambridge, England, pp: 171.
4. BurdfordMason, A.P. and J.M.T. Willoughby, 1986. The efficacy of a new nyastatin formulation in oral candidiasis. Bri. Med. J., 293: 563564.

5. Chakrabarthi A, Ghosh A, Batra R, Kaushal A, Roy P, Singh H. Antifungal Susceptibility pattern of non -Candida albicans and distribution of species isolated from Candidaemia cases over a 5 year period. Indian J Med Res 1996; 104:171-6.

6. Chander J; Textbook of Mycology. Chapter 2, 5, 6, 45, 3rd edition, Mehta publishers, 2009: 9- 20, 35 - 56, 266-290.

7. Jagadish Chander.Candidiasis In: Text Book of Medical Mycology 3rd edn, Mehta Publishers, 2013; 20: 267-283.

8. Koneman E, Allen S, Janda W; K oneman's Colour Atlas and Textbook of diagnostic Microbiology. 5th edition, Lippincott- Raven publishers, 1997: 1043-1047.

9. McGinnis, M.R., 1980. Yeast Identification. In: Laboratory Hand Book of Medical Mycology, McGinnis, M.R. (Ed.). Academic Press, New York, pp: 337411.

10. Mondal S, Mondal A, Pal N, Banerjee p, Kumar S, Bhargava D. Species distribution and In vitro antifungal susceptibility testing pattern of Candida. Journal of Institute of Medicine. 2013; 35:1

11. Nicolatau, O., M. Theodoridou, G. Mostrou, A. Velegraki and N.J. Legakis, 1999. Oral lesions in children with perinatally acquired human immunodeficiency virus infection. J. Oral Pathol. Med., 28: 4953. 
12. Parija SC, Sujatha S, Dhodapkar R; Standard operating procedure manual, Department of Microbiology, JIPMER, Pondicherry, 2011: 37 -42 .

13. Shaheen MA, Taha M; Species identification of Candida isolates from oral lesions of hospitalized and non hospitalized patients with oral candidiasis. Egyptian Derm online Journal, 2006; 2(1): 1-13.

\begin{tabular}{|l|l|}
\hline \multicolumn{2}{|c|}{ Access this Article in Online } \\
\hline & $\begin{array}{l}\text { Website: } \\
\text { www.ijcrims.com }\end{array}$ \\
\hline Quick Response Code & \\
\hline
\end{tabular}

How to cite this article:

R.Venkatajothi. (2017). Prevalence of Oral Thrush Yeasts among School Children with special emphasis of Fluconazole antifungal drug. Int. J. Curr. Res. Med. Sci. 3(3): 125-130.

DOI: http://dx.doi.org/10.22192/ijcrms.2017.03.03.016 